\title{
The Use of Surgical Simulators to Reduce Errors
}

\author{
Marvin P. Fried, Richard Satava, Suzanne Weghorst, \\ Anthony Gallagher, Clarence Sasaki, Douglas Ross, \\ Mika Sinanan, Hernando Cuellar, Jose I. Uribe, \\ Michael Zeltsan, Harman Arora
}

\section{Abstract}

The training of a surgeon includes the acquisition of a number of characteristics. These include a cognitive knowledge base, problem formulation and decisionmaking abilities, appropriate psychosocial relationships, and other attributes that can be measured with objective testing, such as national board or specialty certifying examinations. Perhaps most critical to the surgeon, however, are the core technical skills of the profession. A battery of sophisticated devices is being created to teach and provide objective evaluations of the trainee's technical abilities. These innovative state-of-the-art simulation devices, used to teach basic skills and surgical tasks through repetitive proctored challenges, will enable detection and analysis of surgical errors and near miss incidents without risk to patients. As with flight simulation, near miss detection capabilities anticipate potential errors before they occur and prevent resulting complications. In collaboration with a number of otolaryngology residency programs, we have developed a comprehensive training curriculum, based on complementary simulation tools and several perceptual and spatial ability test instruments, to provide objective metrics for assessing the technical skills of trainees. These tools include an endoscopic sinus surgery simulator for procedural training, and the Minimally Invasive Surgical Trainer-Virtual Reality (MIST-VR) system for basic surgical skills training. Technical errors are identified, quantified, and used to monitor surgical performance, after which the metrics are used for outcome analysis, for the purpose of improving patient safety. Validated metrics include time-to-completion, errors, economy of motion, and psychomotor tracking. Endoscopic sinus surgery is a suitable operation substrate, since it is a frequently performed procedure (more than 300,000 times annually in the United States.) and carries a significant risk of injury to the delicate neighboring eye and brain structures. The endoscopic sinus surgery simulator (ES3) is an advanced surgical simulation device based largely on jet pilot flight simulation, and provides the trainee with appropriate levels of challenge and instruction. ES3 validation studies are reported here, along with a description of the World Wide Web-based Intelligent Information Interface (WI3) simulation database. 


\section{Introduction}

The emphasis on patient safety in the operating room has focused historically on the numerous supportive functions involving devices, medications, staffing, and administrative procedures, but rarely on the surgeon's technique and performance. A surgeon's training occurs during residency and is traditionally a subjective process, as is the assessment of technique and the evaluation of skill, dexterity, and insight. Given that competence and safety are of paramount importance, the objective measure of a resident's progress is a critical factor and one that his or her teachers address diligently.

Otolaryngology residents training in endoscopic sinus surgery (ESS) must learn the anatomy and physiology of the sinuses, the relevant disease processes, and the concepts and skills of surgery. Cadaver dissection may be performed, but most training is conducted in the operating room under the supervision of an experienced surgeon. As training is dependent upon the patients of the attending surgeon, it is imperative that the operating room (OR) environment is kept as riskfree as possible. A high-risk training environment, similar to that encountered by a commercial pilot flying in turbulent weather or by a military pilot flying over hostile territory, cannot be created for the sake of resident education. Excessive intraoperative bleeding would be an analogous example of such a high-risk situation in surgery. Even with the use of cadavers (which don't bleed) for training, once a single dissection is completed, it cannot be "erased" for the purpose of repeating the procedure, since the anatomy has been permanently altered.

Current training of otolaryngology residents in ESS includes videotape viewings, cadaver dissection (where available), and direct observation of procedures performed in the OR. As residents progress in their training, they are given a more active role in the procedure and, in their final year, they become the major participants. For the most part, the technical acumen required for basic ESS procedures is not achieved until the latter half of a resident's training. With the increasing complexity of endoscopic dissection equipment and the ever-increasing demand to broaden the indications for ESS, there is a growing need to make residents more familiar with the technical skills of ESS at an earlier stage in their training.

Optimal visualization is critical to such endoscopic procedures, and the surgeon's understanding of anatomical relationships must be unambiguous and maintained throughout the surgery, even as significant tissue is removed. This anatomical understanding is necessary if the surgeon is to navigate the patient's sinus passages safely and avoid complications. Thus, learning the complex anatomy of the head and the relevant anatomical relationships is essential to ESS, as is the skillful practice of surgical maneuvers.

Computer-generated surgical simulation affords the ability to create an environment that imitates the real surgical world without inherent risk to the patient, and the technology serves to overcome many of the limitations of current training approaches. Procedures can be repeated until proficiency is achieved, 
techniques can be refreshed or learned anew, the variability of case-mix (or lack thereof) can be overcome, and competency can be assessed by certifying boards or hospital accreditation panels, prior to taking a single patient to the operating room. ${ }^{1}$ Moreover, new devices can be tested in a simulated environment, and new procedures can be rehearsed or improved, without exposing patients to potential risk of harm.

Simulation has the potential to improve patient safety and reduce surgical errors by screening would-be surgeons for demonstrable aptitude and providing initial training experience, while at the same time promoting ongoing training with a consistently reproducible process, enabling periodic surgical skill assessments, and cultivating proficiency through rehearsals of complex, patientspecific procedures. ${ }^{2}$

The use of innovative simulation devices that train surgical tasks and basic skills through repetitive proctored challenges may lead to better detection and analysis of potential surgical errors. Error reduction and surgical expertise can be achieved through the implementation of a dynamic, simulation-based curriculum in which objective measurements are acquired and skills are assessed. With exposure to such a curriculum, surgical trainees may be able to achieve a considerable level of prepatient expertise and provide a higher quality of health care during their residency training and beyond.

\section{Role of a surgical simulation}

Virtual reality (VR) simulators based on interactive computer graphics have had a marked impact on the skill level of military and commercial pilots, and may hold similar promise for the medical field. Over the past 3 decades, computerassisted devices have been used to augment the education and training of surgical residents in several fields with significant success. ${ }^{3-8}$ VR simulation already has played an introductory role in training residents for laparoscopic, gastrointestinal, plastic, ophthalmologic, dermatologic, and some laryngologic procedures. ${ }^{7-14} \mathrm{VR}$ simulation's efficacy as a teaching tool is clear, but whether it is superior to conventional teaching methods has yet to be determined. ${ }^{9,10,13}$

A report by Seymour and associates ${ }^{15}$ has begun to address the value of surgical simulation training by demonstrating that VR training has a positive impact on resident performance in the OR, and potentially on safety as well. Similarly, we have developed an integrated training environment using psychomotor, visuo-spatial, and proprioception measurement instruments, along with two sophisticated simulators, to maximize skill acquisition and to gauge trainee progress. One of these simulators is the ES3 (Lockheed Martin, Akron, $\mathrm{OH})$, which permits trainees to progress through a series of increasingly challenging simulation exercises in the course of learning a complete sinus surgery procedure. The other simulator is the Minimally Invasive Surgical Trainer-Virtual Reality (MIST-VR, Mentice Corp., Gothenburg, Sweden), which was developed to train and measure hand-eye coordination and maneuvers analogous to surgical procedures in an abstract environment, using spheres, cubes, 
and virtual surgical instruments. Although these two devices are the state-of-theart, they are indeed rudimentary when one envisions the detail and complexity contained that an ideal surgical environment would possess. Nevertheless, we (and others) are finding them to be useful training tools for teaching many surgical skills.

\section{The endoscopic sinus surgery simulator}

The ESS training simulator was developed in response to the need for a simulator to help train novice sinus surgeons. ${ }^{16,17}$ Computer graphics and haptic (force) feedback are used in the ES3 to create a virtual surgical environment, and the system has been used to teach otolaryngology residents. ${ }^{16-18}$ A team consisting of Lockheed Martin (formerly Loral), the Immersion Corporation, the University of Washington HIT Lab, and the Ohio Supercomputer Center built the ES3 with the sponsorship of the U.S. Army Medical Command and the Telemedicine and Advanced Technology Research Center (TATRC) from 1995 through 1998, furthering the state-of-the-art in surgical simulation.

The ES3 is composed of four principal components: (1) a Silicon Graphics (SGI) computer, which serves as the simulation host platform; (2) a PC-based haptic system controller, which provides the requisite high-rate control over a physical instrument handle associated with a set of virtual surgical instruments; (3) a virtual voice recognition instructor PC, which responds to spoken commands controlling the simulator; and (4) an electro-mechanical platform, which houses a physical replica of an endoscope, the mechanically linked surgical tool handle, and an external head anatomy mannequin. The simulator permits the user to perform endoscopic sinus surgery on a virtual patient, using a wide range of simulated surgical instruments. The surgeon trainee explores the virtual anatomy by manipulating the simulated endoscope in the nose of the mannequin, just as they would with a real patient. With the aid of the virtual instructor PC, the trainee can make audible requests for various virtual instruments and can see them appear subsequently on the monitor. The surgical field appears true-to-life and all instruments, apart from the endoscope, provide haptic feedback through the universal instrument handle. This enables the student to feel tissue resistance appropriate to the tool (i.e., cutting resistance for scissors, vibration for a debrider). ${ }^{19}$

As the student advances through the simulator training, they are given more complex tasks to perform. The student begins training in an abstract "novice" environment, rather than with a virtual patient, and performs tasks designed to familiarize them with the endoscope and the available array of surgical tools. Students learn with the help of predefined navigation path aids, strategically placed targets for injection, and detailed dissection clues. This novice-level training improves hand-eye coordination through an immersive experience. Each task builds upon the skills acquired in the previous task, and all of the tasks relate directly to the complete ESS procedure. Upon achieving an appropriate score at the novice level, the trainee then graduates to the intermediate level, where the 
abstract training aids appear in the context of sinus anatomy and replicate the actual surgical environment. Navigation and injection aids correspond to their novice level counterparts. The system prompts the trainee to perform the appropriate dissection tasks while interacting with labeled anatomical structures. The surgical field appears true-to-life at the advanced level, and the student is expected to perform procedures without the benefit of computer-generated aids. ${ }^{19}$ The virtual instructor points out mistakes, errors, and misses, and can be called upon to suggest the appropriate tool for performing a particular exercise.

The simulator records overall and task-specific scores for each student's performance, including the time needed to complete a task and accuracy relative to optimal performance. A penalty is imposed when excessive time is needed to complete a task, or for hitting "hazards," which represent the anatomical areas the surgeon should take care to avoid. These include the optic nerve and the lamina papyracea. Points are deducted from the trainee's score if one or more hazards are dissected.

\section{Metrics}

How can surgical safety be measured appropriately? Complications are not the sole standard, since mechanisms in current training practices largely prevent their occurrence (primarily via intervention by the attending surgeon). The flight training analogy is very much in order in this regard: A skilled observer assesses the trainee's performance through briefing, debriefing, and over-the-shoulder evaluation, in addition to the use of predetermined metrics, objective simulator responses, and using the trainee's own observations (analogous to the dictated operative note). ${ }^{20-22}$ And while an intentional adverse situation cannot be induced in a patient for the purpose of training or testing the skill of the student, a simulator can achieve this goal with complete safety.

A conference on Metrics for Technical Skills was convened as a component of this research project, with the goal of deriving objective measures for the individual components of an endoscopic sinus operation. Errors in ESS were identified, and each error was classified according to a taxonomy of error types (i.e., technical, cognitive, or combined). The list was approved by a consensus of the conference participants. Quantifiable measures then were defined for each error. Some errors, such as "wrong tool choice," were assigned simple "yes" or "no" measures. Other errors, such as "injury to the lacrimal duct" or "too much scope rotation" have clear and measurable end points. Other errors, such as "past pointing" or "lack of progress," may need further quantification, and an expert rater panel is currently evaluating them with the assistance of recorded procedures. A set of outcome measures was developed with the consensus of the conference participants, to address the broad areas of technical, cognitive, and combined skills, as well as global assessment measures of surgical proficiency. This rigorous process has brought to the project an added emphasis on error reduction prevention as a formal component of surgical training. Students are 
taught in a uniform and comprehensive manner precisely what the errors are and how to avoid them. ${ }^{23}$

The key to error prevention is not so much the detection of errors and related complications, but rather the detection of near misses and the prevention of related errors and complications. One of the strengths of the aviation safety industry's record of performance is the reporting of near misses, which are stressed in simulation training. Because the ES3 can accurately measure the path of the virtual instrument and how close to an anatomical hazard an instrument is placed, it can measure near misses that may or may not result in a postoperative complication. Thus, the ES3 can quantify potential errors in an objective manner, and can better train residents to avoid them by providing near miss detection and feedback.

\section{Current ESS training}

We conducted a survey of the five major centers participating in this research project in an effort to better understand the present state of resident training. The survey asked the program directors 19 questions regarding various aspects of teaching ESS to residents. These include questions such as what year residents began performing ESS; if formal lectures are required prior to performing surgery; what is the curriculum for residents reading prior to surgery; if cadaver dissection is performed and if skills on cadaver dissection is evaluated. Feedback to residents was also queried. The survey yielded the following results: (1) residents often did not begin performing endoscopic sinus surgery until their final year of otolaryngologic training; (2) slightly more than half of the responding institutions gave formal lectures before residents were allowed to perform ESS, and the number of lecture hours varied by institution; (3) slightly more than half of the institutions required the completion of a reading list before residents were allowed to perform in the operating room; and (4) while most training programs afforded their residents the opportunity to practice their ESS techniques on cadaver heads prior to their initial operating room experience, none of the institutions made this experience a requirement. Based upon these results, we have concluded that there is a clear need for an ESS curriculum that is systematic, comprehensive, and well structured, and includes the necessary cognitive content and the surgical skills training.

A comprehensive and integrated curriculum was developed to standardize pretraining for those students taking part in the ES3 simulation studies. This curriculum was compiled on a compact disc (CD), with interactive audio and video information demonstrating successful and erroneous operations at each training level, as well as written background and anatomical information. Preliminary results have shown that this curriculum facilitates to a great extent the trainees' compliance with the simulation training protocols and helps to eliminate bias resulting from variations in the educational backgrounds of our subjects. 


\section{ES3 validation}

Crucial to the project's success is the ability to validate the ES3 as a training tool for endoscopic sinus surgery. One of the studies in our project was designed specifically to address the discriminant and concurrent validity of the ES3. The purpose of this study was two-fold: (1) it compared the performance levels of medical students to that of otolaryngology residents and otolaryngology attending physicians over 23 trials (10 at the novice level, 10 at the intermediate level, and 3 at the advanced level); and (2) it established benchmark data for attending surgeons that can be used to measure resident and medical student simulator performance.

At the Montefiore Medical Center (Bronx, NY), we recruited 38 medical students from the Albert Einstein College of Medicine who received and reviewed the curriculum materials (as discussed above) and completed a demographic survey. Research technicians then gathered performance data on a near-weekly basis for the three ES3 training modes (novice, intermediate, and advanced). Performance data were obtained similarly from 19 residents and 17 attending physicians at the Montefiore and Madigan Army Medical Center (Washington State) simulator sites. New attending and resident data is being added on an ongoing basis.

The data from this study clearly show that there are significant group differences in ES3 simulation training performance by medical students, residents, and attendings. Otolaryngology residents performed significantly better than the medical student group on the novice and intermediate models (Scheffe F $=14.856, P<.05$, and Scheffe $\mathrm{F}=6.236, P<.05$, respectively). Initial performance on the novice level correlated with residency training year and prior ESS experience $(\mathrm{r}=.652, P<.05)$. In support of the simulator's face validity, subject ratings of the virtual model's realism were consistently high on the postsession questionnaire and in open-ended comments. Postsession questionnaire responses also confirm that the otolaryngology residents perceived the simulator as valid and useful ESS training aid.

As one would expect from a simulator that accurately depicts sinus surgery, attending physicians performed the best at all levels, with residents close behind. Although the medical students improved their performance over the course of 10 trials, their performance was substantially beneath that of the residents, when using the performance level of the attendings as the standard (Figure 1). Given the numbers of participants that were enrolled from all of the sites, the results bear substantial statistical power. These findings are unique in that no other benchmark criteria for sinus surgery have been reported previously.

A second study was designed to establish construct validity by examining the correlation between subject performance on the ES3 and respective performance on other validated, objective tests of fundamental visuo-spatial and perceptual abilities. ${ }^{24-28}$ Endoscopic sinus surgery requires simultaneous two-handed coordination of surgical instruments and an endoscope in three-dimensional space. Successful performance of this task requires complex ambidextrous 
Figure 1. Mean ES3 novice level scores for the 3 subject groups across 10 distributed practice trials

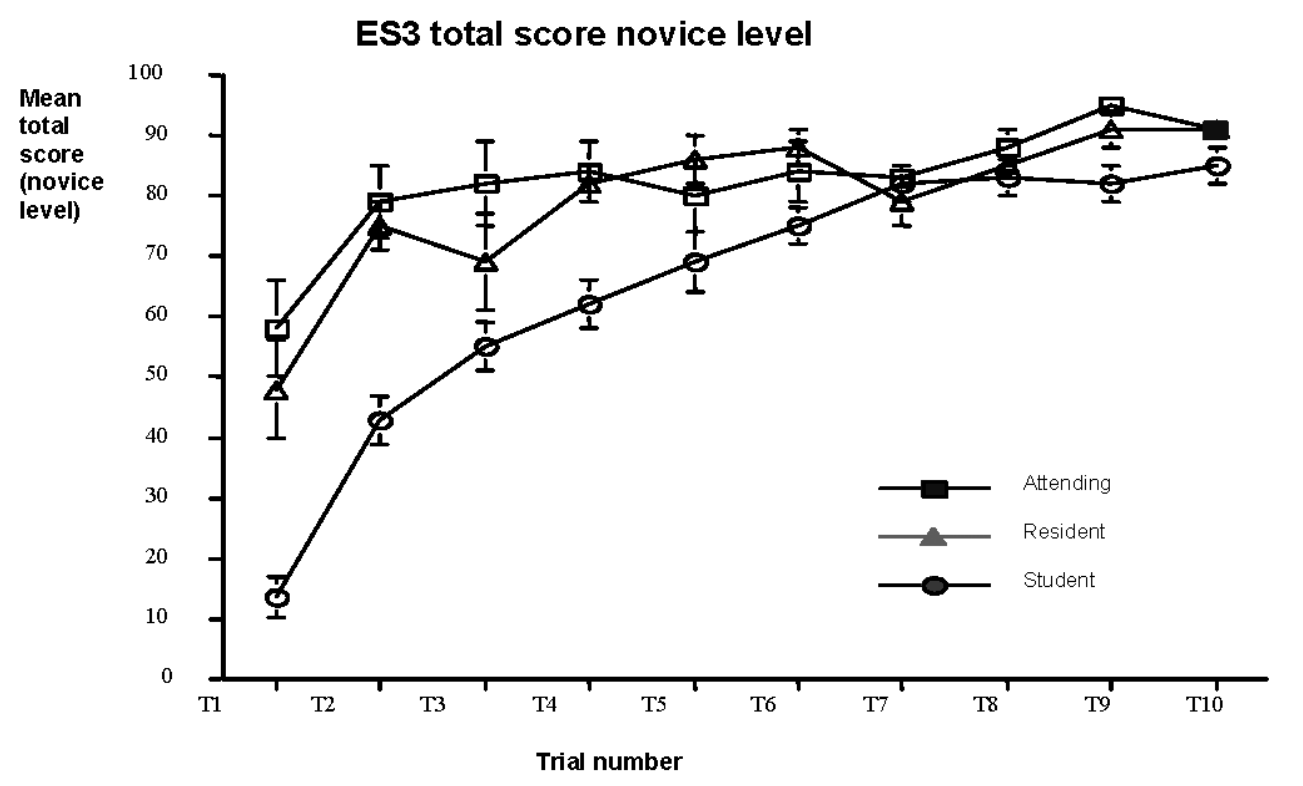

psychomotor, visuo-spatial, and perceptual skill. The goal of this study was to see if objective tests of such abilities, which have already been shown in the literature to predict surgical performance, also might predict performance on the ES3. The subjects of the study were 34 medical students from the Albert Einstein College of Medicine and 4 otolaryngology residents from Montefiore Medical Center.

Results of this study revealed several correlations among these previously validated tests and performance on the ES3 (Table 1). We have shown, through a simple regression model, that scores from (1) the PicSOr (Picture Surface Orientation) test, a validated instrument developed to identify aptitude for extracting information on 3-D structures from 2-D images; (2) the Cube Comparison mental rotation test; and (3) total score on the MIST-VR system, are strong predictors of ES3 performance.

PicSOr scores also have a strong correlation with the ES3 hazard score, a figure that represents the number of errors committed during a simulated surgical procedure. Thus, individual students who have difficulty extracting 3-D information from 2-D images appear to have similar difficulty manipulating a 3-D simulated patient on a standard 2-D monitor.

Similarly, we found that total scores from the MIST-VR trainer show a significant correlation with dissection scores, navigation scores, injection scores, and ES3 total scores, all of which have a strong dependence on hand-eye coordination. It can be concluded then that the ES3 provides an accurate assessment of the technical skills that it proposes to measure, thus providing evidence for the construct validity of this instrument. 
Table 1. Correlations among psychometric and ES3 simulator scores

\begin{tabular}{|l|c|c|c|c|c|}
\hline & EicSOr & $\begin{array}{c}\text { Cube } \\
\text { Comparison } \\
(\text { CC) }\end{array}$ & $\begin{array}{c}\text { Card } \\
\text { rotation } \\
(\text { CR) }\end{array}$ & $\begin{array}{c}\text { MIST-VR } \\
\text { Total score }\end{array}$ & $\begin{array}{c}\text { Regresion } \\
\text { model fit } \\
\text { (ES3 trial } \\
\text { score, PicSOr, } \\
\text { CC, MIST-VR) }\end{array}$ \\
\hline Hazard score & $\begin{array}{c}r=0.5 \\
(P<0.00)\end{array}$ & $\begin{array}{c}r=0.29 \\
(P<0.1)\end{array}$ & $\begin{array}{c}r=0.27 \\
(P<0.14)\end{array}$ & $\begin{array}{c}r=0.36 \\
(P<0.06)\end{array}$ & \\
\hline ES3 trial score & $\begin{array}{c}r=0.3 \\
(P<0.2)\end{array}$ & $\begin{array}{c}r=0.43 \\
(P<0.01)\end{array}$ & $\begin{array}{c}r=0.45 \\
(P<0.01)\end{array}$ & $\begin{array}{c}r=0.57 \\
(P<0.00)\end{array}$ & $\begin{array}{c}r=0.63 \\
(P<0.01)\end{array}$ \\
\hline
\end{tabular}

Significant Pearson correlation coefficients shown in boldface type

\section{VR to OR}

A third study, on the VR-to-OR transfer of training, addresses in a direct manner the question of whether training on the ES3 will improve the endoscopic sinus surgery performance of otolaryngology residents and reduce the incidence of surgical errors. This study requires the collection of resident data from different institutions in East Coast and West Coast programs (Montefiore Medical Center, New York University Medical Center, New York Eye and Ear Infirmary, Columbia University, Yale University, the U.S. Naval Medical Center in San Diego, and Madigan Army Medical Center). Otolaryngology residents in their junior year (e.g., PGY 2 and 3) are assigned either to the experimental group (those receiving conventional sinus surgery training and ES3 training prior to operating on a patient) or a control group (those receiving only conventional sinus surgery training). Individuals in the two groups are videotaped during one of their first five sinus surgery procedures; these tapes then are scored by a select group of trained raters. We have enrolled eight residents from Montefiore Medical Center, thus far, and all eight of them have completed the protocol. Because of the study's complexity and the requirement involving residents in a particular year of their residency, this multi-institutional project is still ongoing. Results, while not yet conclusive, suggest a positive transfer of training from the simulation environment to initial OR procedures.

Previous work by Edmonds during the development of the ES3 demonstrated that surgical confidence correlates highly with dissection skill, suggesting that confidence comes with successful dissection experience. ${ }^{17}$ Simulation training appears to positively affect initial operating room performance, as judged by senior surgeons rating anonymous videotapes of these procedures, with the strongest effects being for instrument manipulation and surgical confidence. ${ }^{17}$ Novice trial scores on the ES3 have been shown to be significant predictors of overall surgical competency. Of the various ES3 performance measures, dissection score on the novice model was found to be the strongest predictor of surgical competency in the OR. Results from a group of nine residents followed a 
consistent trend that suggested transfer of training from the ES3 to several components of surgical competency.

\section{Web-based data repository}

A unique feature of this study is the creation of a World Wide Web-based Intelligent Information Interface (WI3) to serve as a repository for results obtained from remote ES3 simulators located at various institutions. The primary objectives of the WI3 are to provide a centralized server for project documents and data, and an Internet site for analyzing and comparing the results of this project. The database architecture was designed in such a way that it can serve as a template for archiving and accessing results from other surgical simulation projects, as well. The four major components of this architecture are (1) the ES3 simulator computer, which will remain isolated from direct Internet access (to assure confidentiality of trainee scores); (2) the proctoring software, which runs on a PC that is linked directly to the SGI simulation host computer via a local network and with the central database server via the Internet; (3) the central SQL server, which is housed at the University of Washington and manages database input and access functions; and (4) the database access software made available to the general public and the project investigators, via a standard Internet service connection and Web browser interface. The WI3 design is not tied to the ES3 specifically, but is readily generalizable, extensible, and scalable to other surgical (and nonsurgical) simulators.

The system architecture supports three types of simulator trial database access: (1) immediate access to the trainee's percentile score and learning curve, relative to appropriate comparison groups (e.g., senior residents), is available through the proctor's interface during the training trial debriefings - this same information also can be retrieved after the fact through the password-protected Web site server; (2) project investigators can view simple descriptive statistics and data graphics for user-selected database subsets directly from the Web site; and (3) investigators can download user-selected data subsets for further statistical analysis using their PC and standard data analysis software, such as Excel $^{\circledR}$ or SPSS $^{\circledR}$.

In addition to the simulator trial data, the server archives and provides access to various project documents including user manuals, protocol descriptions, data graphics, and drafts of working papers. The server also supports streaming media content, such as videotape samples, and facilitates automated data uploading from the proctor's computer. Without the latter capability, the acquisition and integration of data from remote ES3 sites would be considerably more difficult and more prone to data transfer errors. The remote ES3 systems located on various campuses are essential to achieving adequate subject numbers in a field such as otolaryngology, given that the residency programs are so small. In addition, the WI3 will serve as a prototype for the creation of a national database, whereby each resident's surgical skill can be compared to that of an appropriate 
cohort, as well as to established performance criteria. No such repository for surgical skill data exists to date.

\section{Conclusion}

The use of advanced simulation technology has contributed to an extraordinary aviation industry safety record. Likewise, simulation technology and methodology can be used to improve the quality of surgical training for procedures such as endoscopic sinus surgery (ESS), one of the most common procedures performed by otolaryngologists in the United States - and one that poses significant risk to the surrounding anatomic structures, such as the eye and brain.

We have established a working group that capitalizes on the strengths of numerous investigators and institutions with expertise in the area of otolaryngologic training, image-guided surgery, simulation development, metrics development, and outcome analysis. The overall goal of the collaborative effort is improved patient safety for surgical procedures, using highly sophisticated surgical simulators to improve the screening, education, training, error detection skills, assessment, and certification of surgeons. The resulting technical skill improvements will render safer operative procedures with fewer medical errors.

We anticipate that the next generation of ES3 will generate a virtual surgical environment based on individual patient anatomy data (derived from the patient's own CT scan) that can be downloaded into the simulator. The technology will enable the surgeon to perform a "mission rehearsal" prior to the actual surgery, for the purpose of anticipating risks and hazards, and annotate them with radiology images to be used during the actual surgery. We further anticipate that surgical simulators of the sort described in this paper will achieve a level of performance in the next few years that will make them suitable for surgeon testing and certification.

\section{Acknowledgments}

This study was supported by the Agency for Healthcare Research and Quality, through Grant \# 1 R18 HS11866-01.

\section{Author affiliations}

Department of Otolaryngology, Albert Einstein College of Medicine, Bronx, NY (MPF). Otolaryngology Surgery Simulator Center, Montefiore Medical Center, Bronx, NY (HC, JIU, MZ). Department of Surgery, University of Washington, Seattle, WA (RS, MS). Department of Surgery, Yale University School of Medicine, New Haven, CT (CS, DR). Human Interface Technology Laboratory, University of Washington, Seattle, WA (SW). Emory Endosurgery Unit, Emory University School of Medicine, Atlanta, GA (AG). Albert Einstein College of Medicine, Bronx, NY (HA).

Address correspondence to: Marvin P. Fried, M.D., F.A.C.S., Montefiore Medical Center, 3400 Bainbridge Ave., 3rd Floor, Bronx, NY 10467; phone: 718-920-2991; e-mail: mfried@montefiore.org. 


\section{References}

1. Dawson SL. A critical approach to medical simulation. Bull Am Coll Surg 2002;(87):12-8.

2. Healy GB. The college should be instrumental in adapting simulators to education. Bull Am Coll Surg 2002;(87):10-2.

3. Rosser JC, Herman B, Risucci DA, et al. Effectiveness of a CD-ROM multimedia tutorial in transferring cognitive knowledge essential for laparoscopic skill training. Am J Sur 2000;(179):320-4.

4. Reznick RK. Teaching and testing technical skills. Am J Surg 1993;(165):358-62.

5. Gorman PJ, Meier AH, Krummel TM. Simulation and virtual reality in surgical education: real or unreal? Arch Surg 1999;(134):1203-8.

6. O'Toole RV, Plyter RR, Krummel TM, et al. Measuring and developing suturing technique with a virtual reality simulator. J Am Coll Surg 1999;(189): 114-27.

7. Satava RM. Virtual reality surgical simulator. The first steps. Surg Endosc 1993;(7):203-5.

8. Gorman PJ, Meier AH, Krummel TM. Computerassisted training and learning in surgery. Comput Aided Surg 2000;(5):120-30.

9. Peugnet F, Dubois P, Rouland JF. Virtual reality versus conventional training in retinal photocoagulation: a first clinical assessment. Comput Aided Surg 1998;(3):20-6.

10. Gladstone HB, Raugi GJ, Berg D, et al. Virtual reality for dermatologic surgery: virtually a reality in the $21 \mathrm{st}$ century. J Am Acad Dermatol 2000;(42):106-12.

11. McGovern KT. Applications of virtual reality to surgery. BMJ 1994;(308):1054-5.

12. Satava RM. Virtual endoscopy: diagnosis using 3-D visualization and virtual representation. Surg Endosc 1996;(10):173-4.

13. Baillie J, Evangelou H, Jowell P, et al. The future of endoscopy simulation: a Duke perspective. Endoscopy 1992;(24):542-3.

14. Fried MP, Moharir VM, Shinmoto H, et al. Virtual laryngoscopy. Ann Otol Rhinol Laryngol 1999;108(3): 221-6.

15. Seymour NE, Gallagher AG, Roman SA, et al. Virtual reality training improves operating room performance: results of a randomized, double-blinded study. Ann Surg 2002;(236):458-63; discussion 463-4.

16. Rudman DT, Stredney D, Sessanna D, et al. Functional endoscopic sinus surgery training simulator. Laryngoscope 1998;(108):1643-7.
17. Edmonds CV, Heskamp D, Sluis D, et al. ENT endoscopic surgical training simulator. In: Morgan KS, editor. Medicine meets virtual reality. Amsterdam: IOS Press; 1997. pp. 518-28.

18. Wiet GJ, Yagel R, Stredney D, et al. A volumetric approach to virtual simulation of functional endoscopic sinus surgery. Stud Health Technol Inform 1997;(39):167-79.

19. Edmonds CV, Heskamp D, Sluis D, et al. ENT endoscopic surgical training simulator. Stud Health Technol Inform 1997;(39):518-28.

20. Nullmeyer RT, Rockway MR. Effectiveness of the C130 weapon system trainer for tactical aircrew training. Proceedings of the 4th Interservice/ Industry Training Simulation and Education Conference, Washington, DC; 1984. National Training Systems Association. Arlington, VA.

21. Nullmeyer RT, Crane P, Cicero G, et al. A bridge between cockpit/crew resource management and distributed mission training for fighter pilots. Proceedings of the 21st Interservice / Industry Training Simulation and Education Conference, Orlando FL; 2000. National Training Systems Association. Arlington, VA.

22. Bills C, Butterbodt VL. Total training systems design to function a total quality management application. Proceedings of the National Aerospace Electronics Conference (NAECON), Dayton, OH; 1992 May 1822. IEEE Aerospace and Electronics Systems Society.

23. Satava RM, Fried MP. A methodology for objective assessment of errors: an example using an endoscopic sinus surgery simulator. Otolaryngol Clin N Am 2002; (35):1289-1301.

24. Gallagher AG, Cowie R, Crothers I, et al. PicSOr: an objective test of perceptual skill that predicts laparoscopic technical skill in three initial studies of laparoscopic performance. Surg Endosc 2003;(17): 1468-71.

25. Gallagher AG, Crothers I, Satava RM. Objective measures of visuo-spatial ability for minimally invasive surgery. Presented at the European Association for Endoscopic Surgery, Barcelona, Spain; 2004.

26. Hamilton EC, Scott DJ, Fleming JB, et al. Comparison of video trainer and virtual reality training systems on acquisition of laparoscopic skills. Surg Endosc 2002; (16):406-11.

27. Gallagher AG, McGuigan J, Ritchie K, et al. Objective psychomotor assessment of senior, junior and novice laparoscopists with virtual reality. World J Surg 2001; (25):1478-83. 
28. Gallagher AG, Satava, RM. Objective assessment of experience, junior and novice laparoscopic

performance with virtual reality: learning curves and reliability measures. Surg Endosc 2002;(16):1746-52. 
